\title{
OTP based Lineman Security System
}

\author{
Manohara H. C ${ }^{1, *}$, Dr. Gopiya Naik. $\mathrm{S}^{2}$, Tejaswni K. $\mathrm{P}^{3}$, Shana C. $\mathrm{M}^{3}$, Kowsalya. S ${ }^{3}$, Meghana. R N \\ ${ }^{1}$ Assiastant Professor, \\ ${ }^{2}$ Associate Professor, ${ }^{3}$ Students, \\ Department of EEE. PESCE, Mandya, Karnataka (India)
}

\begin{abstract}
The main motive of this work is to lower the rate of problems incurred during fault conditions of lines \& lamp; other maintenance work carried out by line man. The electric line man safety system makes use of a new concept of one-time password (OTP). It is found that the fatal electrical accidents to the line man are increasing during the electrical line repair due to lack of communication and co-ordination between the maintenance staff and electric substation. This proposed system provides a solution that ensures the safety of maintenance staff, i.e., line man. On detecting a fault in electric line, the line man sends SMS to deactivate the line and which is again activated after completing the work. It can also prove a boon to save power, thus it saves the life of line man working on electric line. The proposed system is fully operated by microcontroller.
\end{abstract}

Keywords: Line man, Security, OTP, Microcontroller, GSM module.

\section{INTRODUCTION}

\subsection{Lineman Security}

Electricity is hazardous: an electric shock from a current as low as $35 \mathrm{~mA}$ is sufficient to cause fibrillation of the heart in the vulnerable individuals. Even a healthy individual is at risk of falling from a high structure due to loss of muscle control. Higher currents can cause respiratory failure and result in extensive and life- threatening burns. The lack of any visible sign that a conductor is energized even at high voltages, makes electricity a particular hazard. At high voltages it is unnecessary to come into direct contact with charged equipment to be shocked. An electric field surrounds all the charged devices. Bringing a conducting object such as a human into that field can intensify the field enough for electrical breakdown of the air and an arc to jump from the equipment to earth via that person.

A line man, power line technician is a tradesperson who constructs and maintains electric power transmission, telecommunication lines (a cable, internet and phone) and distribution lines. Because of this hazardous nature of electricity the security for line man must be provided to ensure his safety. A line worker generally does outdoor installations and maintenance jobs. Those who install and maintain electrical wiring inside buildings are electricians. Safety is the prime concern in our day to day life. Everyone needs to be secure as much as possible. There are many electrical accidents happened and still happening due to lack of security provided to the lineman. Recently, in Kunigal of Tumkur district (Karnataka), a lineman named Swamy died while operating on transmission line because of the lack of security. The electric line man safety system is designed to control a circuit breaker by using a password for the safety of electric man. Nowadays, electrical accidents to the line man are increasing, while repairing the electrical lines due to the lack of communication between the electrical substation and maintenance staff.

This work gives a solution to this problem to ensure line man safety. In this system the control (ON/OFF) of the electrical lines lies with line man. The main objective of this work is to save life of line man by making such a protective system controlled through SMS. In this proposed system if there is any fault in line, the lineman sends the password due to which main line is switched off, then he works on line to repair and again resends SMS to switch on the electrical line. Components which are used in our model reduces the time which is required for the line man for repairing and are easily available in the market. The main concept of our project is to save time of working for the line man and also to safeguard his life. The main part of our project is the GSM module which is required for sending a SMS.

\subsection{Literature Survey}

In order to revise the problem objectives literature survey has been conducted. So many papers are collected, out of which three are presented and drawbacks are identified. Efforts have been made to overcome this drawbacks.

\subsubsection{Password Based Circuit Breaker}

In this method Microcontroller are used which controls all the operations in regarding the password system. For this process we require the components like microcontroller control circuitry, power supply and key pad. These keypads are used for entering password for operating different load which are connected to the controller. If suppose password is wrong, then load will not be switched to the controller and then the controller checks for the precaution instruction which is provided by the developer. This includes the operations such as the number of loads to be opened, the number of threshold levels that are crossed. In this process the controller checks the number of threshold levels that are crossed and according to that the gates are being controlled. The drawbacks of this method are: There is chance of forgetting the password. The line man has to go for the power station for setting up the password.

1.2.2 User Changeable Password Based Circuit Breaker

A circuit breaker is an automatically operated electrical switch designed to protect an electrical circuit from damage caused by overload or short circuit. Its basic function is to detect a fault condition and interrupt current flow. Unlike a fuse, which operates once and then must be replaced, a 
circuit breaker can be reset (either manually or automatically) to resume normal operation. When operated manually we see fatal electrical accidents to the line man are increasing during the electric line repair due to the lack of communication and coordination between the maintenance staff and the electric substation staff. In order to avoid such accidents, the breaker can be so designed such that only authorized person can operate it with a password. Here, there is also a provision of changing the password. The system is fully controlled by the 8-bit microcontroller of 8051 family. The password is stored in an EEPROM, interfaced to the microcontroller and the password can be changed any time unlike a fixed one burnt permanently on to the microcontroller. A keypad is used to enter the password and a relay to open or close circuit breaker, which is indicated by a lamp. Any wrong attempt to open the breaker (by entering the wrong password) an alert will be actuated, indicated by another lamp. The drawbacks of this method are: The line man has to go for the power station for setting up the password.

\subsubsection{Circuit Breaker Based on Android:}

This method provides a password based circuit breaker system using an android application. Here we connect the circuit with android application through which we enter the password. There is a substantial increase in the number of fatal accidents involving line men due to electric shocks resulting from the lack of coordination between maintenance staff and the electric substation staff. This system provides a solution to this problem, to ensure there are no such incidents that endanger the life of line men. Here the control of the circuit is provided at the substation. The line man can enter the password to switch OFF the circuit.

\subsection{Problem Statement}

Here in all the existing methods, lineman needs to visit the power station in order to set up the password and there is a chance of forgetting the password. Even in android method, it is only applicable to Bluetooth range. Hence in order to overcome this problem we are proposing a new method known as "OTP based lineman security system". Here we use GSM and microcontroller as a main component to generate and store one time password respectively. Like in mobile communication, GSM module helps to communicate lineman with the power station to control the lines. Hence this avoids electrical accidents and to save the life of the lineman.

\subsection{Objective}

1. The main objective of this project is to save line man by making such a protective system controlled through one time password.

2. The previous techniques produces manual errors, with the help of password the proposed system provides solution that ensures safety.

\section{PROPOSED METHODOLOGY}

\subsection{Block Diagram}

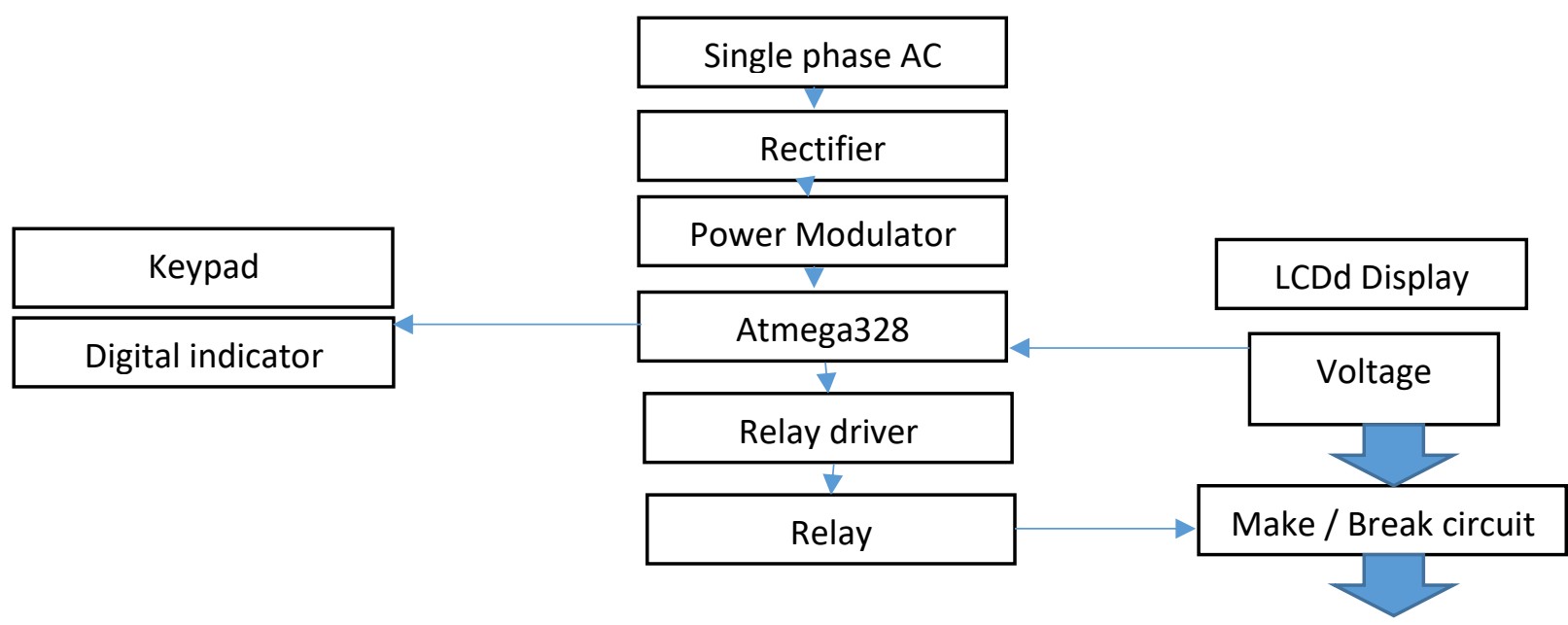

Fig 1: Architectural block diagram of proposed system.

\subsection{Working Principle}

The flowchart in Fig.1 illustrates Architectural block diagram of proposed "OTP based lineman security system". In this proposed system whenever the lineman wants to take line clear (LC), he will send a OTP which is usually less than 256 as we are using 8 bit micro controller. We designed a system in such a way where line man sends OTP in a format 'Q123DCT1'. This format represents a OTP where DCT stands for deactivation, 1 stands for feeder number and 123 is a 3 digit password that a lineman wants to generate.
Similarly in order to activate the feeder, lineman needs to send the same password in the form 'Q123ACT1'. Here the three digit number must be same in order to activate the deactivated line. When the lineman send this information in such a format as a SMS to the power station where actually GSM module is placed, the main authority will get another message at the same time about the details regarding the latest LC in order to avoid problem in case when the lineman forgets the password and also to store the details. Here without the main authority permission, lineman cannot 
operate this function. Even though if he tries to do line charge without informing higher authority illegally, He will get caught because the high authority's (AEE) number will be fed in the program so they will be having his complete information regarding line charge.

\subsection{Functional Block Diagram of Microcontroller}

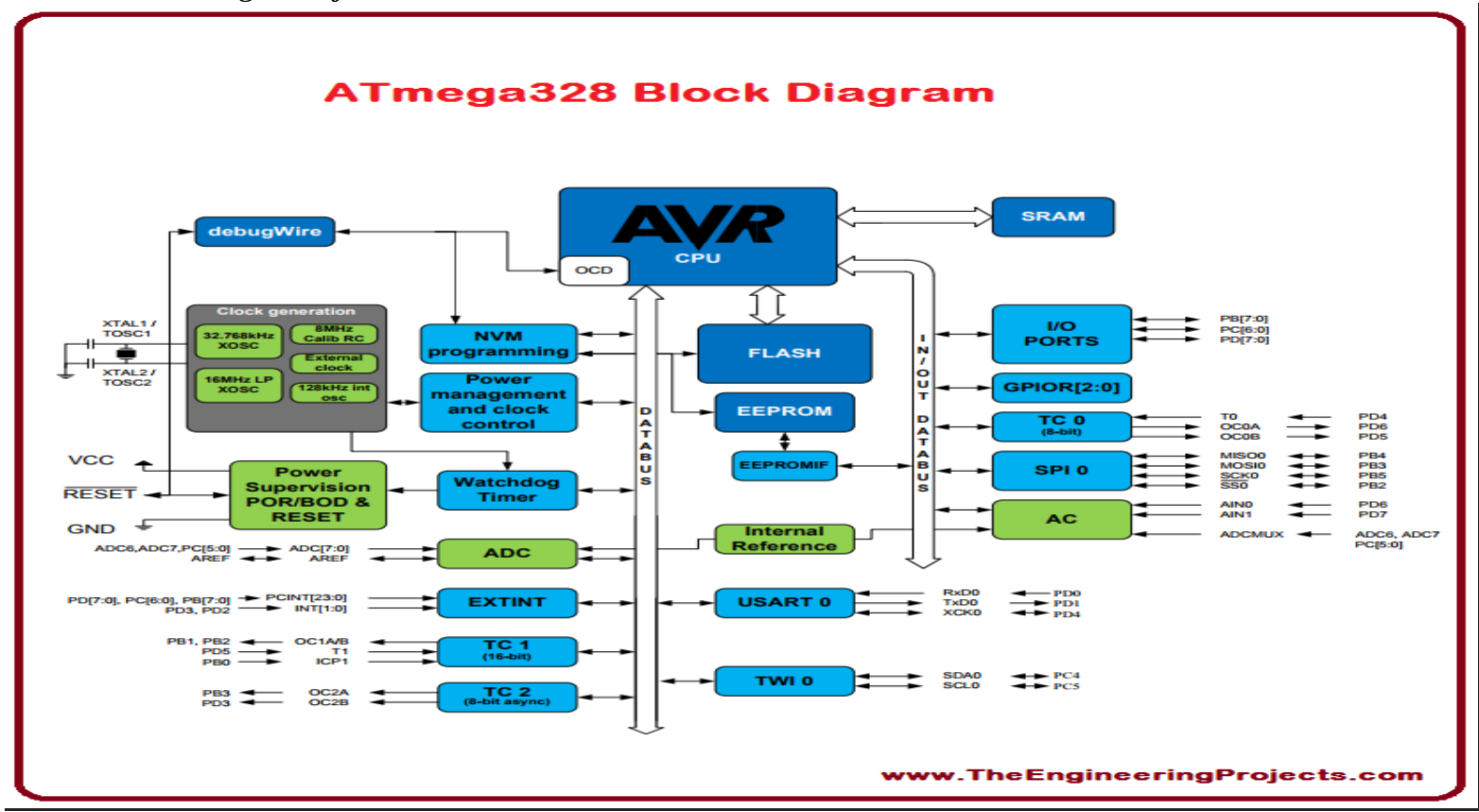

2.4. Flow Chart of Proposed System

Fig. 2: Functional block diagram of ATMEGA-328.

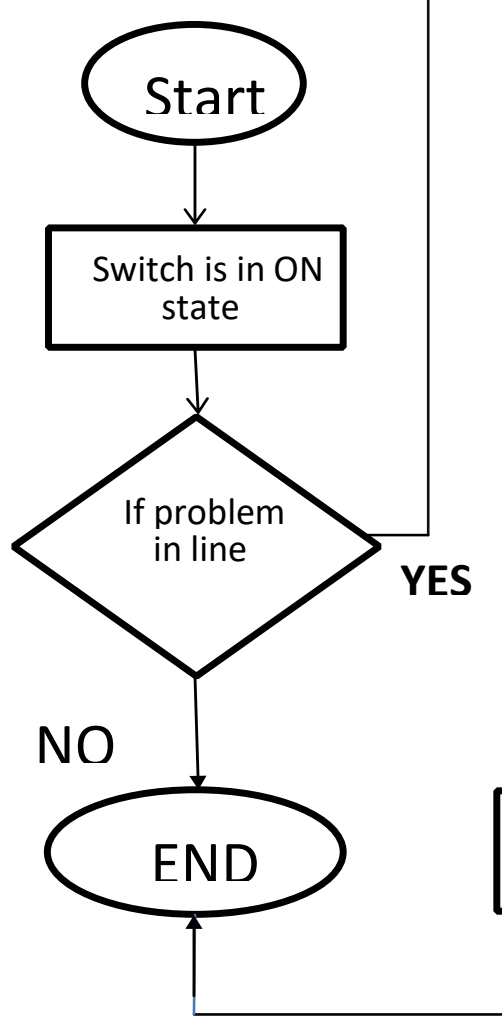

Make a TEXT (OTP)

power circuit

Micro controller

gets interrupt

LCD Disblav shows

circuit is off in nower station

Fig. 3: Flowchart of the proposed system. 


\section{RESULTS AND DISCUSSION}

Figure 4 shows the practical setup of the proposed lineman security system based on OTP.

This work can be used to ensure the safety of the maintenance staff, eg. Lineman. The line can be only turned off/on by the line man. This system provides an arrangement such that a password is required to operate the circuit breaker (0N/OFF). Lineman can turn off the supply and comfortably repair it, and then turn on the line by entering the correct password. Since it has the provision of entering the password through registered mobile, only authorized person can enter the password. In case of remote areas where signal is unavailable password can be entered through keypad. Higher authority also has a record of password and lineman operating status. In the cases of lineman forgetting the password, he can consult the authority.

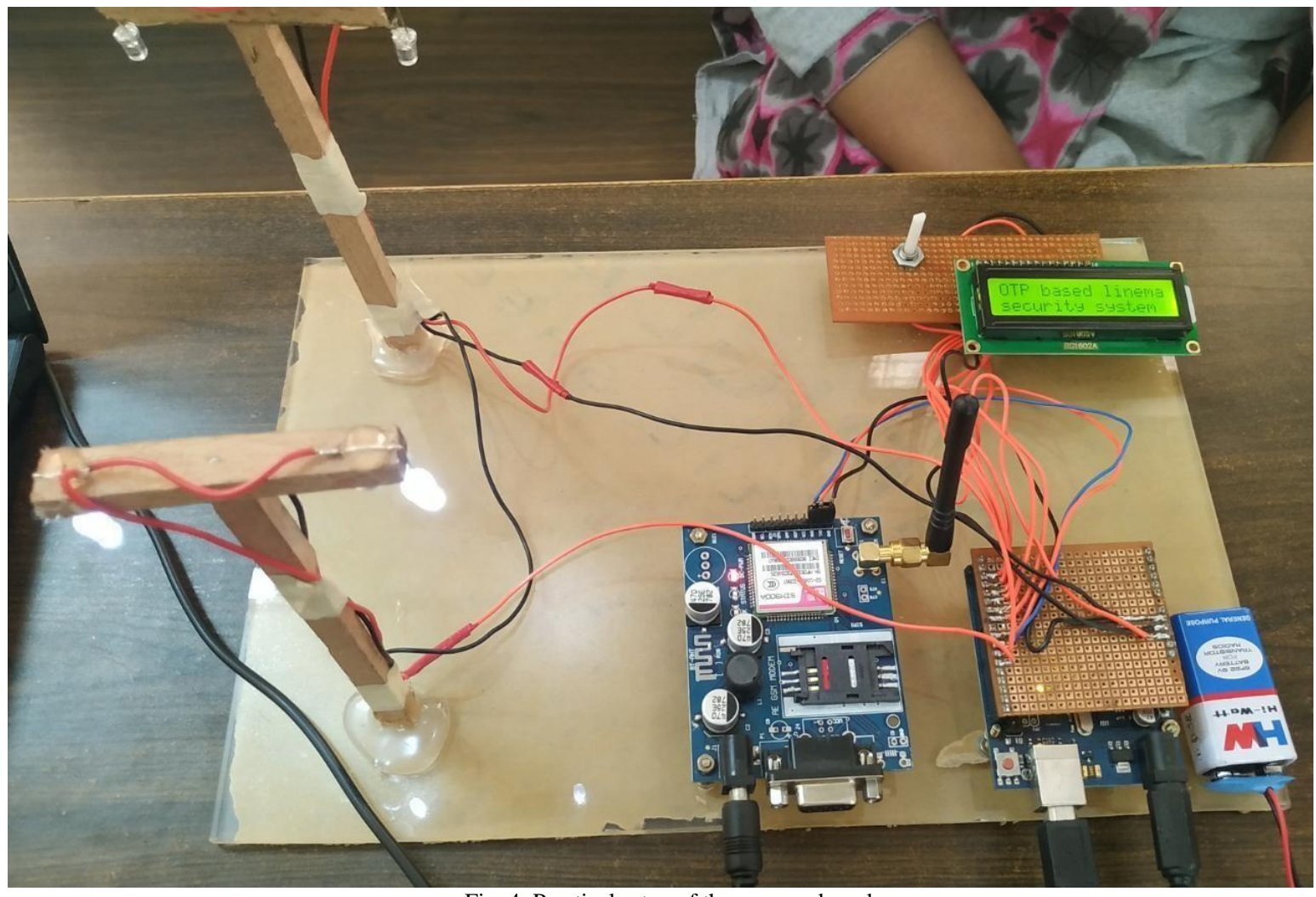

Fig. 4: Practical setup of the proposed work.

\section{CONCLUSION}

The implementation of this work gives an idea of security, thus the proposed system can be used to maintain one time password that cannot be stolen. The control over power supply is maintained continuously. It can be used with SCADA system to atomize the operation and enhance the security. Supervisory control and data acquisition (SCADA) is a control system so SCADA can also be implemented to know where the fault occurs in the system directly and so the lineman can directly locate the fault location and can rectify it. We can also use EEPROM that can be interface to the system so the circuit breaker can not only operate in substation but also from the distance through wireless communication. This work can be interfaced with the GSM module for the remote control of the circuit breaker via SMS.

\section{ACKNOWLEDGEMENT}

The authors would like to express the deepest thanks to the principal, PESCE, Mandya, and the Management, PET(R), Mandya, for their timely support and constant encouragement in successful completion of this research work.

\section{REFERENCES}

[1] Mane Kirti M, et al., "Password Based Circuit Breaker", International Journal for Research in Applied Science \& Engineering Technology (IJRASET), Volume 6 Issue IV, April 2018.

[2] Sebin J Olickal, "Electric line man safety system with one time password based circuit breaker", IJRET, Vol. 4, No. 3, April 2015 .

[3] Narendra Khandelwal, Tanuj Manglani, Ganpat Singh, Amith kumar, Dilip Khatri, "Automated load distribution with password protected circuit breakers", International journal of recent research and review", Vol. 8, No. 1, March 2015.

[4] National electrical safety code committee, accredited standards committee C2 rule 441 Table 441-1 AC live work minimum approach distance (2002 edition, page-228) IR540.

[5] National electrical safety code, 1997 edition

[6] ATMEGA 328/P data sheets. 


\section{BIBLIOGRAPHY}

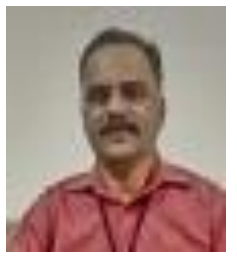

MANOHARA H.C. completed his B.E. in Electrical and Electronics Engg. in the year 2000 at S.J College of Engineering, Mysore (University of Mysore) and M.Tech in VLSI \& Embedded system Design from VTU, Belgaum in the year 2008. From 2000 to 2010 he has served as a lecturer in EEE department at SJCE, Mysore. At present he is working as an Asst. Prof. in the Dept. of E\&E, PESCE, Mandya, since 2010. His area of interests are Power system Protection and renewable energy system.

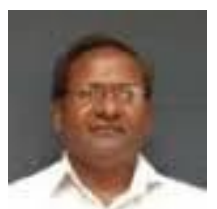

Gopiya Naik. S completed his BE degree in Electrical and Electronics from MCE, Hassan, M.Tech in power system from NIE, Mysore and Ph.D from IIT, Roorkee. He has got total 24 years of teaching experience and published many papers in the national and international journals of repute. At present he is working as an associate professor in the dept. of Electrical and Electronics Engineering at PESCE, Mandya, Karnataka. His research interest includes: power distribution system planning, renewable energy systems-grid integration, micro grid, digital integrated circuits in electronic devices and robotics and applications. 